\title{
DLX3 regulates bone mass by targeting genes supporting osteoblast differentiation and mineral homeostasis in vivo
}

\author{
J Isaac ${ }^{1}$, J Erthal ${ }^{1}$, J Gordon², O Duverger ${ }^{1}$, H-W Sun ${ }^{3}$, AC Lichtler ${ }^{4}$, GS Stein ${ }^{2}$, JB Lian² and MI Morasso*,1
}

Human mutations and in vitro studies indicate that DLX3 has a crucial function in bone development, however, the in vivo role of DLX3 in endochondral ossification has not been established. Here, we identify DLX3 as a central attenuator of adult bone mass in the appendicular skeleton. Dynamic bone formation, histologic and micro-computed tomography analyses demonstrate that in vivo DLX3 conditional loss of function in mesenchymal cells (Prx1-Cre) and osteoblasts (OCN-Cre) results in increased bone mass accrual observed as early as 2 weeks that remains elevated throughout the lifespan owing to increased osteoblast activity and increased expression of bone matrix genes. DIx3OCN-conditional knockout mice have more trabeculae that extend deeper in the medullary cavity and thicker cortical bone with an increased mineral apposition rate, decreased bone mineral density and increased cortical porosity. Trabecular TRAP staining and site-specific Q-PCR demonstrated that osteoclastic resorption remained normal on trabecular bone, whereas cortical bone exhibited altered osteoclast patterning on the periosteal surface associated with high Opg/Rankl ratios. Using RNA sequencing and chromatin immunoprecipitation-Seq analyses, we demonstrate that DLX3 regulates transcription factors crucial for bone formation such as D/X5,DIx6, Runx2 and Sp7 as well as genes important to mineral deposition (Ibsp, Enpp1, Mepe) and bone turnover (Opg). Furthermore, with the removal of DLX3, we observe increased occupancy of DLX5, as well as increased and earlier occupancy of RUNX2 on the bone-specific osteocalcin promoter. Together, these findings provide novel insight into mechanisms by which DLX3 attenuates bone mass accrual to support bone homeostasis by osteogenic gene pathway regulation.

Cell Death and Differentiation (2014) 21, 1365-1376; doi:10.1038/cdd.2014.82; published online 20 June 2014

Endochondral bone formation (EBF) and homeostasis require a regulated program of mesenchymal condensation, chondrocyte differentiation, vascular invasion, cartilage resorption, osteoprogenitor recruitment and differentiation and bone remodeling. These key processes are under the control of essential bone transcription factors (TFs), including RUNX2, SP7 and homeodomain protein families such as HOX, MSX and DLX. ${ }^{1-3}$ DLX proteins are important regulators of developmental and differentiation processes, including skeletal development. ${ }^{4-6}$ A missense mutation in $D L X 5$ leads to split hand and foot malformation ${ }^{7}$ and DLX5 and DLX6 are positive transcriptional regulators of osteochondroblastic differentiation. ${ }^{6}$ DLX3 is defined as an osteogenic regulator, as human mutations in $D L X 3$ lead to tricho-dento-osseous (TDO) syndrome, an ectodermal dysplasia that causes increased bone mineral density (BMD) in intramembranous and endochondral bones. ${ }^{8}$ In vitro, osteocalcin (Ocn), Runx $2^{9,10}$ and osteoactivin ${ }^{9-11}$ are directly regulated by $\mathrm{DLX} 3$, and overexpression of $\mathrm{DLX} 3$ in osteoprogenitors stimulates transcription of osteogenic markers. ${ }^{9}$

Recently, we investigated the effects of neural crest deletion of $D / x 3$ in craniofacial bones. ${ }^{12}$ The gene signature of craniofacial bones from Wnt1cre:D/x3 neonates predicted increased bone formation and mineralization. This was further supported by ex vivo assays on frontal bone osteoblasts, suggesting an inhibitory role for DLX3 in osteoblastic differentiation. ${ }^{12}$ Contrary to this prediction, adult mice exhibited decreased BMD and increased porosity in neural crest-derived craniofacial bones. ${ }^{12}$

A transgenic mouse model expressing the TDO DLX3 gene mutation driven by the osteoblast-specific 2.3 Col1A1 promoter was characterized by Choi et al. ${ }^{13}$ This model resulted in increased trabecular bone volume in young and adult mice; however, the diaphysis phenotype was not described. Despite bone marrow stromal cells (BMSCs) from mutants exhibiting enhanced osteoblastic differentiation and increased bone marker expression ex vivo, the dynamic bone

\footnotetext{
'Laboratory of Skin Biology, NIAMS, NIH, Bethesda, MD, USA; ${ }^{2}$ Department of Biochemistry, University of Vermont, Burlington, VT, USA; ${ }^{3}$ Biodata Mining and Discovery Section, NIAMS, NIH, Bethesda, MD, USA and ${ }^{4}$ Department of Genetics and Developmental Biology, University of Connecticut Health Center, Farmington, CT, USA

*Corresponding author: MI Morasso, Laboratory of Skin Biology, NIAMS/NIH, Building 50, Room 1523, Bethesda, 20892 MD, USA. Tel: +1 3014357842 ; Fax: +1 301435 7910; E-mail: morasso@ nih.gov

Abbreviations: Adv, adenovirus; ALPL, alkaline phosphatase; BMD, bone mineral density; BMSC, bone marrow stromal cell; ChIP, chromatin immunoprecipitation; cKO, conditional knockout; CTX-1, carboxy-terminal type I collagen crosslink; DLX3, distal-less homeobox 3; EBF, endochondral bone formation; ECM, extracellular matrix; H\&E, hematoxylin and eosin; MAR, mineral apposition rate; M-BMM, M-CSF-dependent bone marrow macrophages; $\mu \mathrm{CT}$, micro-computed tomography; OCN, osteocalcin; OPG, osteoprotegerin; RANKL, receptor activator of nuclear factor kappa-B ligand; TDO, tricho-dento-osseous; TF, transcription factor; TRAP, tartrate-resistant acid phosphatase; WT, wild type

Received 27.11.13; revised 25.4.14; accepted 07.5.14; Edited by G Melino; published online 20.6.14
} 
formation rate was not increased in vivo and the trabecular phenotype was attributed to decreased osteoclast formation and bone resorption activity due to the increased serum levels of IFN- $\gamma \cdot{ }^{13}$

Taken together, these data highlight an important role for DLX3 in bone; however, the studies above ${ }^{12,13}$ provide contrasting results and emphasize that the function of DLX3 in the postnatal and adult skeleton has not yet been fully elucidated. To address the in vivo role of DLX3 in osteoblastogenesis, bone density, and remodeling in the appendicular skeleton, we generated conditional knockouts (cKOs) of $D / x 3$ in mesenchymal cells $\left(D / x 3^{\text {Prx1-cKO }}\right)$ and in osteogenic lineage cells $\left(D / \times 3^{O C N-c K O}\right)$. In both models, DIx $3^{C K O}$ mice experienced a significant increase in bone mass accrual throughout their lifespan associated with enhanced osteoblast activity. By combining in vivo gene profiling and ex vivo cellular analyses, we establish a newly defined role of DLX3 as a major regulator of bone apposition and homeostasis.

\section{Results}

Deletion of DIx3 in osteogenic lineage cells leads to increased bone mass accrual. High levels of DLX3 were found in osteoprogenitor cells, bone-forming osteoblasts and matrix-embedded osteocytes in both endochondral developing bones and the postnatal skeleton (Figure 1). To address DLX3 function in osteoblastogenesis, DIX3 was deleted in mesenchymal cells using Prx1-cre mice and in osteogenic lineage cells with $O C N$-cre mice, resulting in $D / x 3^{P r \times 1-c K O}$ and $D / x 3^{O C N-c K O}$ mice. We validated the temporal and tissuespecific expression of the Cre transgenes (Supplementary Figure S1). Deletion of D/x3 was confirmed by Q-PCR and western blot (Figures 2a-c). Endochondral bones of $D / \times 3^{O C N-c K O}$ neonates showed no obvious defects in developmental patterning (missing or transformed bone) or gross abnormalities in mineral deposition (P0.5 and P2.5; Supplementary Figure S2).

With micro-computed tomography $(\mu \mathrm{CT})$ analysis, we quantified structural parameters of femurs from 2 weeks (wk) to 6 months (mo) male mice. At 5 weeks, D/x3 $3^{O C N-C K O}$ mice showed a striking phenotype of an overall increase in trabecular and cortical bone mass that continued throughout adult life (Figure 2, Table 1). Although no significant difference was observed in the length of femurs, 3D reconstruction showed an increased length of the trabecular bone area that extended into D/x3 $3^{O C N-c K O}$ diaphysis (Figures $2 \mathrm{~d}$ and e). Across age groups, as early as 2 weeks and maintained as late as 6 months, an increase of trabecular bone volume and number of trabeculae in D/x $3^{O C N-C K O}$ mice was observed (Figure $2 \mathrm{~h}$, Table 1). The increased number of trabeculae and connectivity was complemented by decreased trabecular spacing. However, the thickness and BMD (a measurement of mineral content/unit area) of individual trabeculae remained similar to $D / x 3^{+/+}$mice, a reflection of normal mineralized bone matrix being produced by osteoblast lineage cells in $D / \times 3^{O C N-c K O}$ mice. These results indicate that $D / \times 3^{O C N-C K O}$ osteoprogenitors form more trabeculae that extend deeper into the medullary cavity.

Cortical bone quantification showed increased thickness in $D / x 3^{O C N-C K O}$ mice due to a significant increase of the sub-periosteal area, a rich source of osteoprogenitor cells (Figures $2 \mathrm{f}-\mathrm{h}$, Table 1). Dynamic bone formation was assessed by calcein injections in $5 \mathrm{wk}$ mice. The mineral apposition rate (MAR) was significantly increased on the periosteal surface of the DIx $3^{O C N-c K O}$ tibias $(3.67 \pm 0.18 \mu \mathrm{m} /$ day and $2.2 \pm 0.12 \mu \mathrm{m} /$ day, respectively $(P<0.001))$. Calcein labeling of the cortical surfaces further demonstrated an increased bone apposition rate (distance between two calcein bands) in $D / x 3^{O C N-c K O}$ mice, which is an osteoblast-mediated process (Figure $2 \mathrm{~g}$ ). An intense irregular calcein-labeling pattern was observed around trabeculae, indicative of increased metaphyseal bone remodeling in young mice (Figure $2 \mathrm{~g}$ ). However, DIX3 ${ }^{\mathrm{OCN}-\mathrm{CKO}}$ cortical bone also showed increased porosity and decreased BMD (Figures 2f-h, Table 1). As predicted from Prx1-cre transgene expression (Supplementary Figure S1), DIx3 $3^{\text {Prx1-cKO }}$ mice exhibited a strikingly similar phenotype to $D / x 3^{O C N-c K O}$ mice with increased trabecular bone formation, increased thickness, decreased BMD and increased porosity in cortical bone (Supplementary Figure S3).

In younger $D / x 3^{O C N-c K O}$ mice ( 2 and $5 \mathrm{wk}$ ), there is highly active bone formation and remodeling to convert woven bone to lamellar bone and this is reflected in the porosity of the cortical bone. The \% porosity in the femur decreased from a fold-change (DIx $3^{O C N-c K O} /$ wild type (WT)) of 1.6 to 1.4 to 1.2 at 2 wk, 5 wk and 4 mo, respectively (Table 1). Thus, the small marrow spaces within the cortical bone observed at $5 \mathrm{wk}$ (Figure $3 \mathrm{~A}$ ) are there to support continued remodeling and viability of a larger mineralized bone area, as shown by hematoxylin and eosin (H\&E) and tartrate-resistant acid phosphatase (TRAP) stainings (Figures 3 and 4). Therefore, the decreased porosity in $D / x 3^{O C N-C K O}$ mice with age suggests that cortical bone has matured into adulthood.

Increased osteoblast activity and remodeling underlie the DIX $3^{O C N-c K O}$ phenotype. H\&E staining of tibia sections from 5 wk mice confirmed $\mu \mathrm{CT}$ results showing increased trabecular density and revealed blood vessels in thickened $D / x 3^{O C N-C K O}$ cortical bones (Figure $3 \mathrm{~A}$, insert 4). Primary spongiosa trabeculae (region in close proximity to the growth plate) and cortical bones were densely lined with alkaline phosphatase (ALPL)-positive cells, supporting that the increase in bone formation resulted from increased osteoblast activity (Figure 3B).

To investigate bone resorption, serum levels of carboxyterminal type I collagen crosslink (CTX-1), a biomarker of osteoclastic activity were assessed and showed no significant difference between $D / \times 3^{+/+}$and $D / \times 3^{O C N-C K O}$ mice (Figure 4A). TRAP staining was examined to localize osteoclast cells in long bones (Figures 4A and B). TRAPpositive osteoclast counts and $\operatorname{TRAP}(+)$ surface normalized by bone matrix surface showed no significant difference between $\mathrm{D} / \mathrm{x} 3^{+/+}$and $\mathrm{D} / \mathrm{x} \mathrm{3}^{\mathrm{OCN}-\mathrm{cKO}}$ trabeculae (Figure $\left.4 \mathrm{~A}\right)$. In the diaphysis, however, while osteoclast count and $\operatorname{TRAP}(+)$ surface were not significantly affected in $D / \times 3^{O C N-C K O}$ mice (Figure 4A), histological analysis revealed a variation in osteoclast localization as $D / \times 3^{O C N}-c K O$ bone matured in the diaphysis region (Figure $4 \mathrm{~B}$ ). Although $D / \times 3^{+/+}$mice showed resorptive pit areas along the length of the periosteal surface of the cortical bone, DIx $3^{O C N-c K O}$ mice showed few 


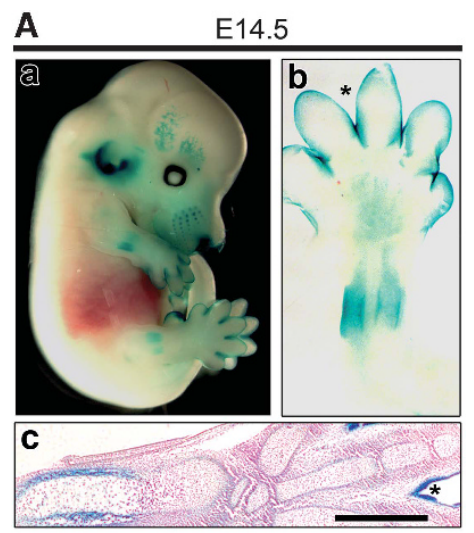

\section{B E16.5}
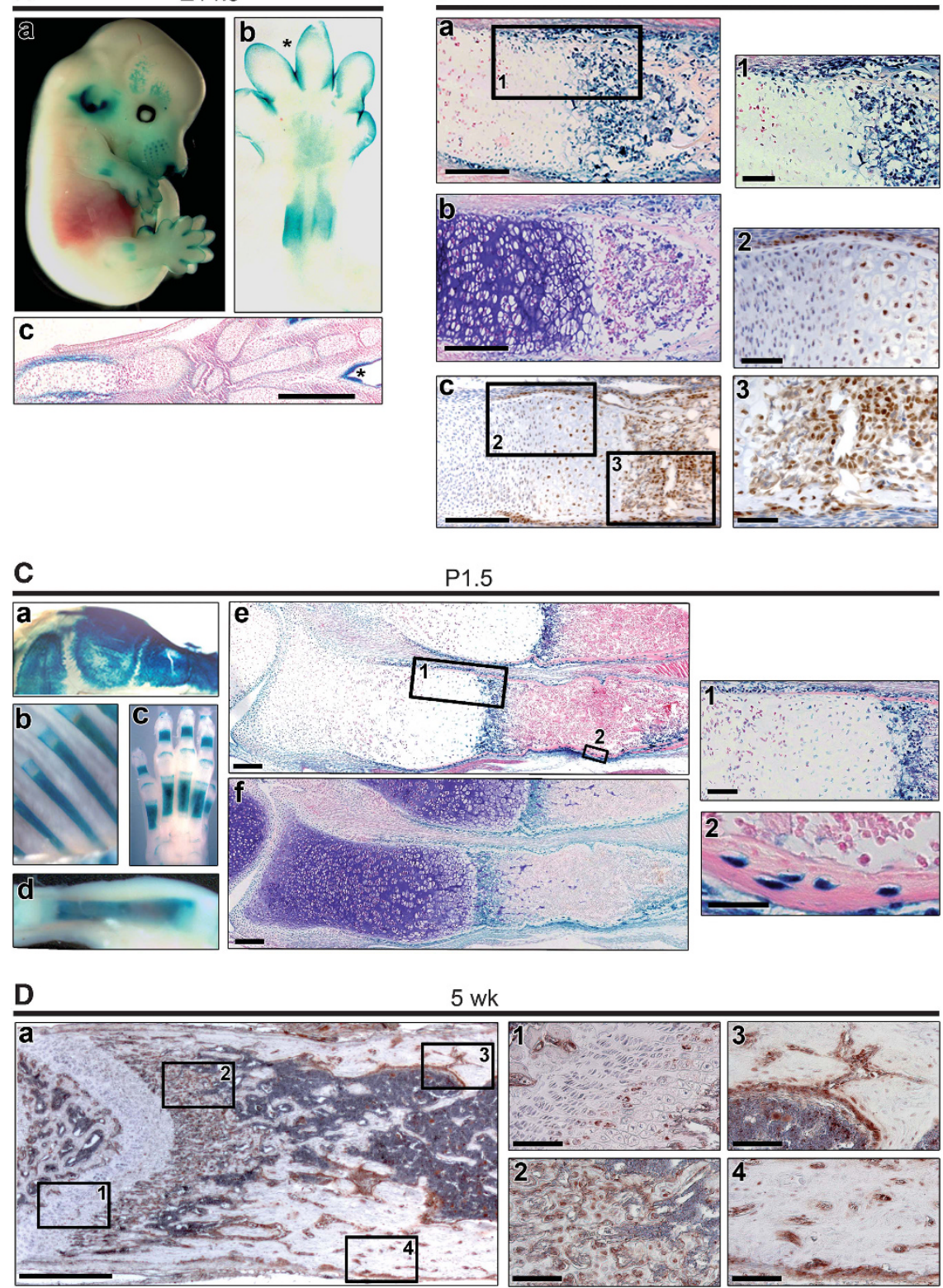

5 wk

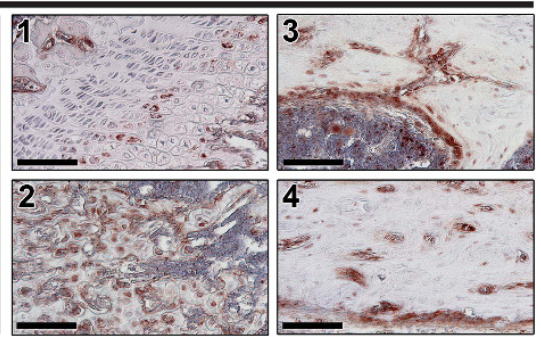

Figure 1 Temporal and spatial Dlx3 expression during bone development. (A) DLX3 localization at E14.5 was shown by LacZ expression in D/x $3^{+/ K i n L a c Z}$ embryos using whole-X-gal staining (a). DLX3 expression in bone collar is shown in benzyl-benzoate-cleared X-gal-stained forelimb (b) and in longitudinal section of the radius (c). Asterisk: LacZ showed DLX3 was expressed in skin as has previously been reported. ${ }^{48}$ (B) DLX3 expression at E16.5 was shown by LacZ detection in longitudinal sections of $D / x 3^{+/ K i n L a c Z}$ humeri (blue staining) (a, insert 1) coupled with toluidine blue staining to visualize the cartilage matrix (purple staining) (b). Immunohistochemistry was performed

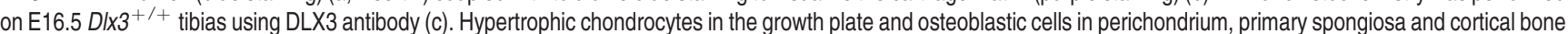
are shown in higher magnification in (c, inserts 2 and 3). (C) DLX3 localization in P1.5 D/x3+/KinLacZ mouse shown by whole-X-gal staining in benzyl-benzoate-cleared calvaria (a), ribs (b), manus (c) and tibia (d). Longitudinal sections of the X-gal-stained tibia (e) coupled with toluidine blue staining (f). Hypertrophic chondrocytes in growth plate and osteoblastic cells in perichondrium, primary spongiosa and cortical bone are shown in higher magnification in (C:e, inserts 1 and 2). (D) DLX3 protein expression is detected at 5 wk by immunohistochemistry with DLX3 antibody on $D / \times 3^{+/+}$tibia (a). Higher magnifications showed hypertrophic chondrocytes in the metaphysis (D, insert 1), active surface osteoblasts in the trabecular bone area (a, insert 2), endosteal (a, insert 3) and periosteal (a, insert 4) surfaces of the diaphysis, and osteocytes in the cortical bone (a, insert 4). Scale bars: $100 \mu \mathrm{m}$ for the main images (letters), $20 \mu \mathrm{m}$ for the inserts (numbers). Eosin was used as counterstaining in X-gal-stained sections and hematoxylin was used for immunochemistry

TRAP-positive cells on the periosteal surface, whereas notable TRAP staining was detected on the endosteal surface and in vascular channels (Figure 4B). However, in cortical bone surrounding the metaphysis region (Figure 4B), TRAPstained osteoclasts were evident on the periosteal and endosteal surfaces of both controls and mutants.
To determine whether the altered osteoclast localization in $D / x 3^{O C N-C K O}$ diaphysis was due to cell autonomous defects in osteoclastogenesis, ex vivo M-CSF-dependent mononuclear cells isolated from bone marrow of $D / x 3^{O C N-c K O}$ mice were cultured (Figures 4C and D). Absence of D/x3 expression in osteoclasts corroborated previous reports. ${ }^{14}$ Furthermore, no 

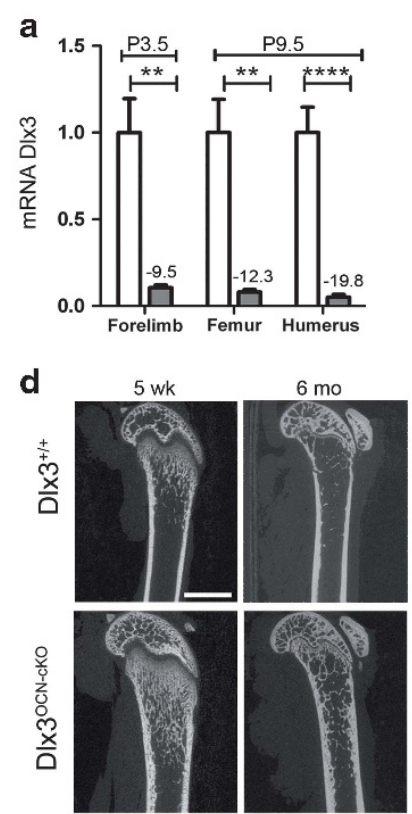

b
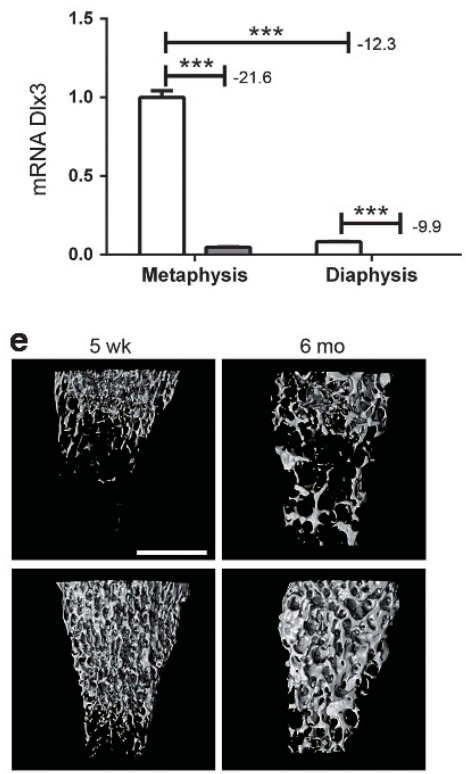
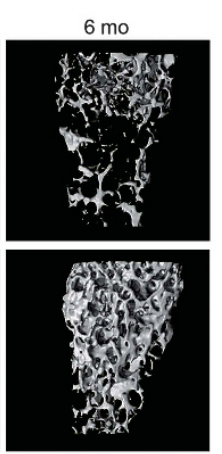

c
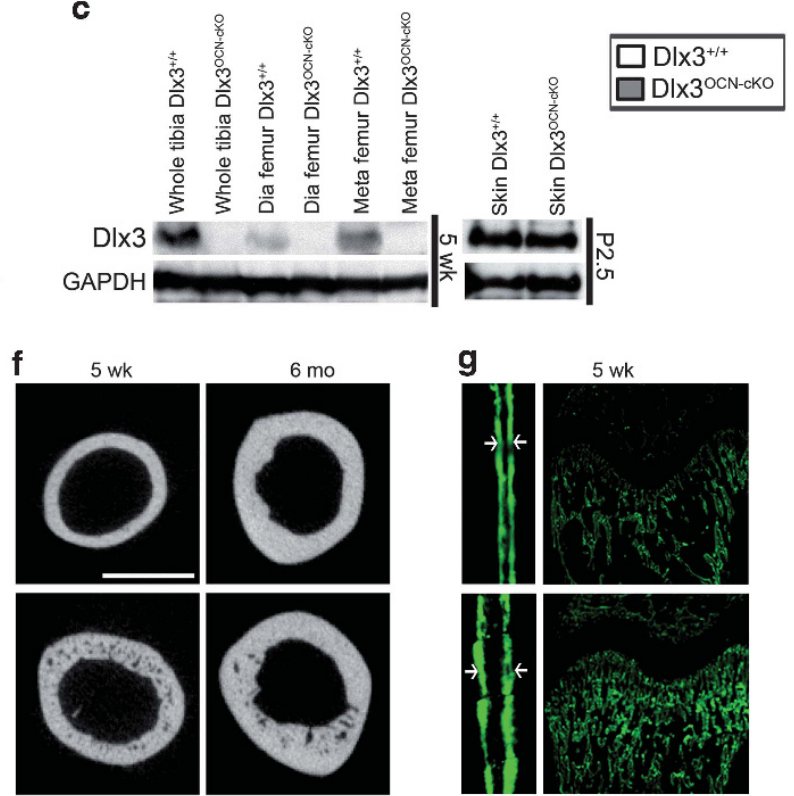
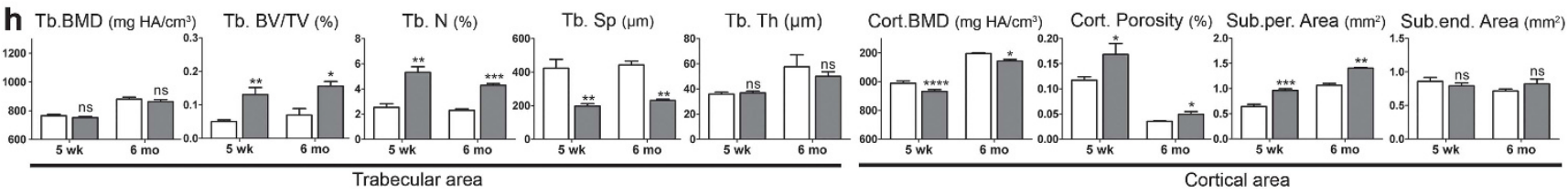

Figure 2 Altered bone formation in DIX3 ${ }^{\mathrm{OCN}-\mathrm{CKO}}$ mice. (a) Q-PCR of DIx3 in long bones of P3.5 and P9.5, (b) and in metaphysis (Meta) and diaphysis (Dia) of 5 wk $D / \times 3^{O C N-c K O}$ and DIX3 ${ }^{+/+}$mice. (c) Western blot of DLX3 in tibia, Dia and Meta of 5 wk mice. P2.5 skin was control for bone-specific deletion. Sagittal femur $\mu C T$ (d), $3 \mathrm{D}$ trabecular reconstructions (e) and transverse scans at mid-diaphysis (f) in 5 wk and $6 \mathrm{mo} D / \times 3^{+/+}$and $D / \times 3^{\mathrm{OCN}-c K O}$ males. (g) Calcein labeling of cortical (left) and trabecular (right) tibia of 5 wk $D / \times 3^{+/+}$and $D / \times 3^{\mathrm{OCN}-c K O}$ males. Scale bars: $1 \mathrm{~mm}$. (h) $\mu \mathrm{CT}$ parameters. Trabecular BMD (Tb. BMD), bone volume ratio (Tb. BV/TV), number (Tb. N), spacing (Tb. Sp) and thickness (Tb. Th) were calculated as were cortical bone mineral density (Cort. BMD), porosity and sub-periosteal and sub-endosteal areas. Data are presented as the mean \pm S.E.M. ns: non-significant, ${ }^{\star} P<0.05,{ }^{* *} P<0.01,{ }^{* * \star} P<0.001,{ }^{* * *} P<0.001$. Scale bars: $1 \mathrm{~mm}$

visible differences in TRAP staining or Trap mRNA levels were observed between $\mathrm{D} / \mathrm{x} 3^{\mathrm{OCN}-\mathrm{CKO}}$ - and $\mathrm{D} / \mathrm{x} 3^{+/+}$-derived cells differentiated into multinucleated osteoclasts (Figures $4 \mathrm{C}$ and $\mathrm{D}$ ). Altogether, these findings were consistent with the CTX-1 and TRAP quantification results (Figure 4A), and therefore confirmed that alteration in osteoclast localization did not result from osteoclast-cell autonomous defects.

Expression of genes associated with signaling between osteoblasts and osteoclasts was evaluated by Q-PCR (Figures 4E and F). The metaphysis of femurs of $5 \mathrm{wk}$ $D / \times 3^{O C N}-c K O$ and $D / x 3^{+/+}$males showed no significant difference in mRNA expression of Mcsf (Csf1), RankI (Tnfs11) and Opg (Tnfrsf11b) or in the ratio of Opg/Rankl expression (index of osteoclast remodeling). These results were in line with the metaphyseal osteoclast counts and indicated that the increased D/x $3^{O C N-c K O}$ trabecular bone volume did not arise from decreased osteoclast number or activity. In contrast, $D / \times 3^{O C N}-c K O$ diaphysis showed increased Mcsf mRNA, significantly increased Opg, and a high Opg/Rankl ratio when compared with controls (Figures $4 \mathrm{E}$ and F). These results indicated that the alteration in osteoclast localization was associated with the variation in expression of osteoclastogenesis markers in cortical bone and suggested that the decreased osteoclasts on the active bone-forming sub-periosteum region of $\mathrm{Dlx} 3^{\mathrm{OCN}-\mathrm{cKO}}$ diaphysis might result from enhanced secretion from osteoblasts of the osteoclast inhibitor osteoprotegerin (OPG).

RNA sequencing (RNA-Seq) and chromatin immunoprecipitation (ChIP)-Seq of DLX3-deficient bones identify DLX3 molecular targets that regulate bone mass. To understand the DLX3-dependent molecular mechanisms involved in EBF, a global gene profiling in metaphysis and diaphysis of D/x $3^{O C N-c K O}$ femurs was performed using RNASeq and significantly affected genes (fold-change (FC) \pm 1.85 , $\mathrm{q}<0.05$ ) were analyzed (Figure 5; Supplementary Figure S4). Data sets have been added to the GEO database (GSE53105). DIx3 ${ }^{O C N-c K O}$ metaphyses and diaphyses showed upregulation of Emilin3, a regulator of mesenchymal commitment and osteoprogenitor differentiation, ${ }^{15}$ as well as upregulation of late stage-osteoblast regulators of mineralization and calcium phosphate balance such as $V d r^{16}$ and Enpp1. ${ }^{17}$ Genes encoding enzymes that promote extracellular matrix turnover such as Adamts 15 and 18 were also upregulated. The enhanced expression of these enzymes was consistent with remodeling of newly formed bone to mature bone.

In the metaphysis, Alpl and the SIBLING genes Ibsp and Spp1 that are associated with bone formation, matrix mineralization and remodeling ${ }^{18,19}$ were upregulated 
(Figure 5a). Other upregulated genes included TFs crucial for the regulation of osteoblast differentiation and ossification, such as Runx2, Sp7, DIx5 and DIx6 (Figure 5a) $)^{1,3}$ and for long-bone development and patterning such as the Hox gene family members HoxA3/B2/B3/B4 (Supplementary Figure S5A). ${ }^{20}$ In $D / \times 3^{O C N-c K O}$ diaphysis, genes related to bone mass were upregulated, particularly Mepe, a SIBLING member and regulator of bone formation and mineralization. ${ }^{21}$

Table 1 Summary table of histomorphometric bone parameters in femurs from

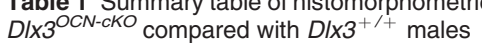

\begin{tabular}{|c|c|c|c|c|}
\hline \multirow[t]{2}{*}{ Mut versus WT (FC) } & \multicolumn{4}{|c|}{$\mathrm{DIx} 3^{\mathrm{OCN}-\mathrm{cKO}} / \mathrm{WT}$} \\
\hline & 2 wk & 5 wk & $4 \mathrm{mo}$ & $6 \mathrm{mo}$ \\
\hline $\begin{array}{l}\text { Femur BMD } \\
\text { Trabecular parameters }\end{array}$ & -1.1 & -1.1 & 1.0 & 1.0 \\
\hline Tb. BMD & 1.0 & 1.0 & 1.0 & 1.0 \\
\hline Tb. BV/TV & 4.3 & 2.6 & 1.8 & 2.2 \\
\hline Tb. number & 1.7 & 2.1 & 1.7 & 1.9 \\
\hline Tb. spacing & -1.7 & -2.1 & -1.8 & -1.9 \\
\hline Connec. density & 58.8 & 5.0 & 2.9 & 6.6 \\
\hline Tb. thick. & 1.3 & 1.0 & 1.0 & -1.2 \\
\hline Cortical parameters & & & & \\
\hline Cort BMD & -1.1 & -1.1 & 1.0 & 1.0 \\
\hline Cortical porosity & 1.6 & 1.4 & 1.2 & 1.4 \\
\hline Sub-periosteal area & -1.2 & 1.5 & 1.5 & 1.3 \\
\hline Sub-endosteal area & -1.1 & -1.1 & 1.2 & 1.2 \\
\hline
\end{tabular}

Quantification of bone parameters in total femur area, trabecular area (Tb.) and mid-diaphysis area (Cort.) in femurs from DIx3 ${ }^{\mathrm{OCN}-\mathrm{cKO}}$ in comparison with Dlx $3^{+/+}(\mathrm{WT})$ males at 2 weeks, 5 weeks, 4 months and 6 months. Trabecular bone mineral density (BMD), bone volume ratio (BV/TV), number, spacing, connectivity (Connect.) density and thickness (Thick.) were calculated in the trabecular area. Cortical BMD, porosity and sub-periosteal and sub-endosteal areas were calculated in the mid-diaphysis area. Data were calculated with at least two animals per condition and expressed in FC of the mutants in comparison with the controls
Finally, genes encoding proteins associated with keysignaling pathways for bone development, such as WNT, TGFb/BMP and NOTCH signaling were enriched in $D / x 3^{O C N-C K O}$ femurs (Supplementary Figure S5B). ${ }^{22-25}$ To investigate if the aforementioned genes are targets of a DLX3regulatory network during EBF and homeostasis, a ChIP-Seq profile of osteoblasts cultured from bone marrow was performed. Our results showed that DLX3 was bound to the promoter of D/x5,D/x6 and $S p 7$, and multiple regions of the Ibsp, Enpp1, Adamts18 and Opg genes, supporting direct regulation of these genes by DLX3 in osteoblasts (Figure $5 \mathrm{c}$ ).

\section{Ex vivo differentiation of osteoprogenitors supports that} DLX3 functions as an attenuator of osteoblastogenesis. To confirm that DLX3 regulates osteoblasts in a stagespecific, osteoblast cell autonomous fashion, calvarial osteoblasts and BMSCs from long bones were isolated from $D / x 3^{F / F}$ neonates and adolescents, respectively, and infected with adenovirus (Adv) containing GFP (Adv-GFP) or Cre recombinase (Adv-Cre) (Figure 6; Supplementary Figure S6). Q-PCR and western Blot showed DIx3 was efficiently excised in Adv-Cre cells (Figures 6a and b; Supplementary Figure S6A). DLX3 deletion in both cell models resulted in increased ALPL activity and, in calvarial cells resulted in higher cell autonomous mineralization capacity (Figure 6c; Supplementary Figure S6B). Expression of osteoblast differentiation and maturation markers $A / p l$ and $I b s p$ was increased in Adv-Cre calvarial cells and BMSCs at later time points (Figure 6d; Supplementary Figure S6C). DIx3 deletion in BMSCs was also associated with significantly increased expression of the TFs Runx2 and DIx5 and the regulator of matrix mineralization Enpp1 (Supplementary Figure S6C). Our results show that $D / x 3$ deletion in osteoblasts results in an enhancement of osteoblast differentiation analogous to the in vivo DIX3 ${ }^{O C N-c K O}$ phenotype.
A

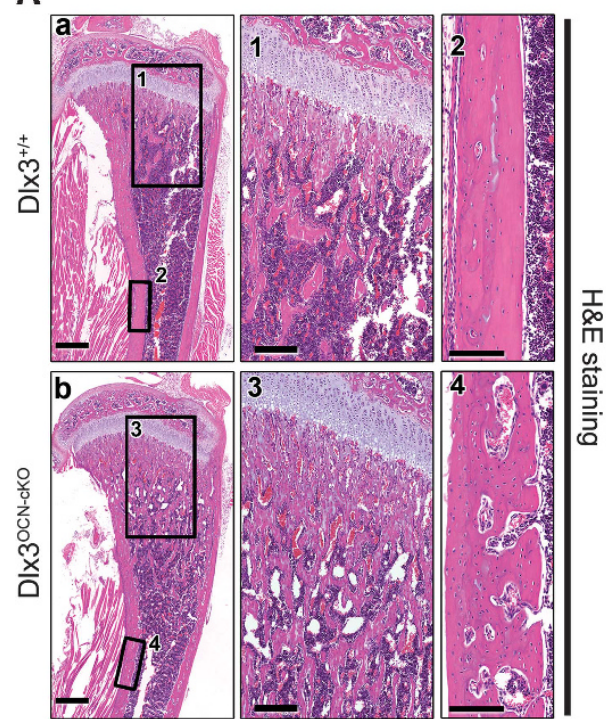

B

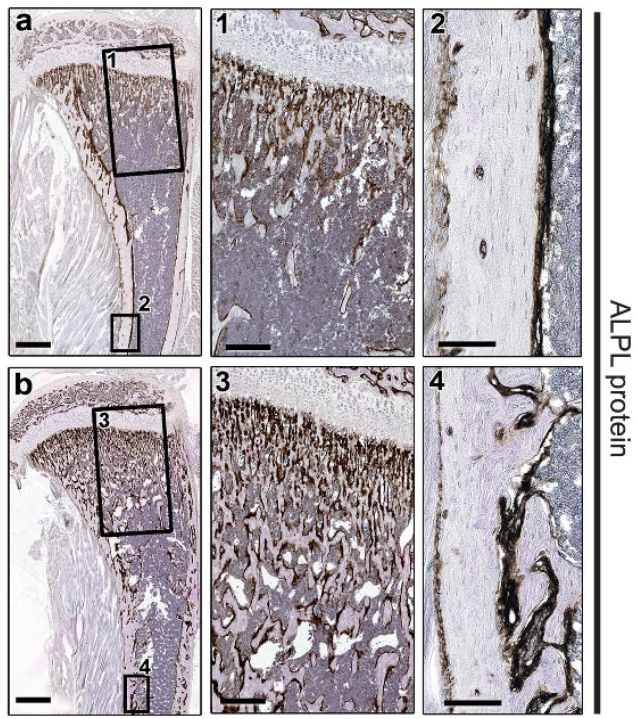

Figure 3 In vivo effects of D/x3 deletion in osteogenic lineage cells on bone formation. Paraffin sections of decalcified tibias of 5 wk D/x3 ${ }^{+/+}$and D/x $3^{O C N-c K O}$ males stained with hematoxylin and eosin (A). Osteoblast localization and activity are shown with ALPL antibody (B) on decalcified tibias. Metaphysis and diaphysis are shown in high magnification (inserts 1, 3 and inserts 2, 4, respectively). Scale bars: $500 \mu \mathrm{m}$ for the main figures (letters), $200 \mu \mathrm{m}$ for the inserts (numbers) 
A
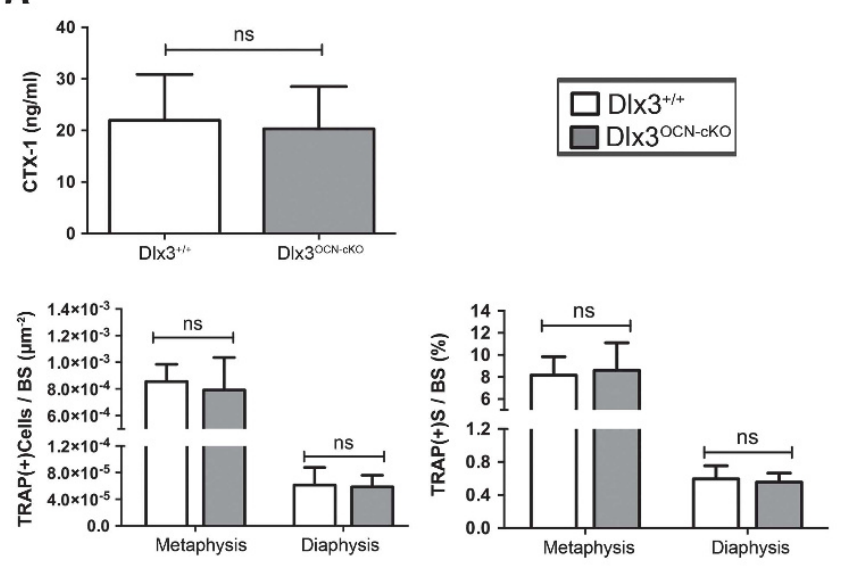

B

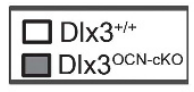

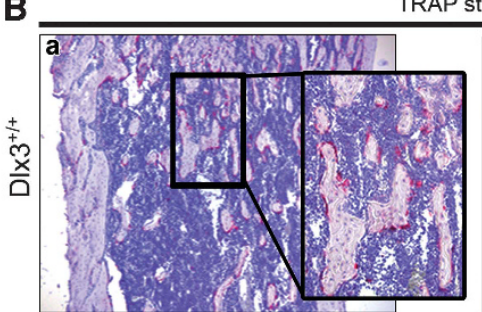

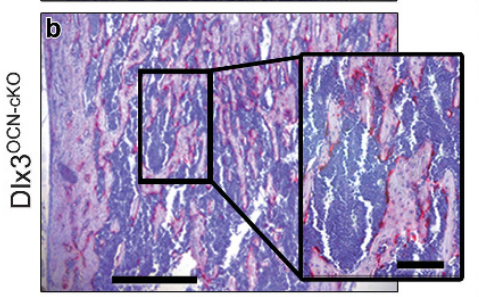

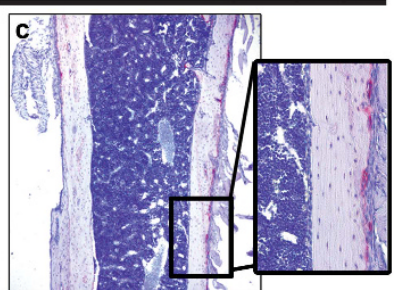

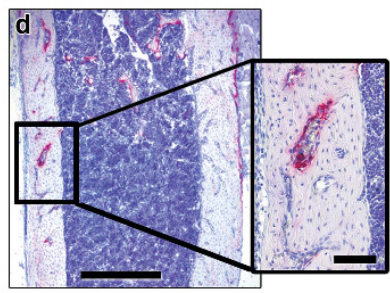

C

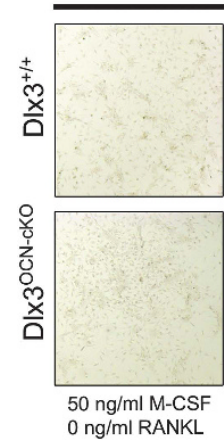

D6 TRAP staining

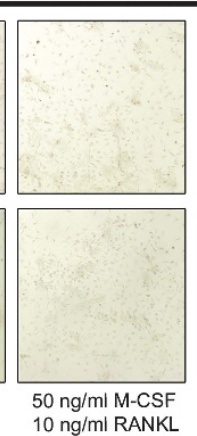

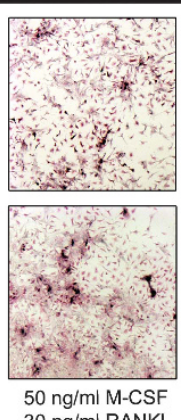

$30 \mathrm{ng} / \mathrm{ml}$ RANKL
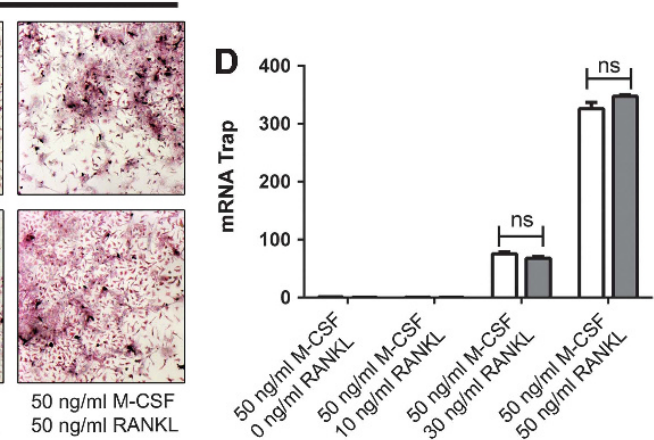

E

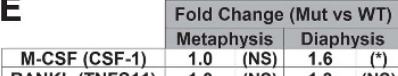
\begin{tabular}{|c|cc|cc|}
\hline M-CSF (CSF-1) & 1.0 & (NS) & 1.6 & (*) \\
\hline RANKL (TNFS11) & 1.0 & (NS) & 1.3 & (NS) \\
\hline
\end{tabular} \begin{tabular}{|l|ll|lc|}
\hline RANKL (TNFS11) & 1.0 & (NS) & 1.3 & (NS) \\
\hline OPG (TNFRSF11B) & 1.1 & (NS) & 3.5 & $\left.{ }^{*}\right)$
\end{tabular}

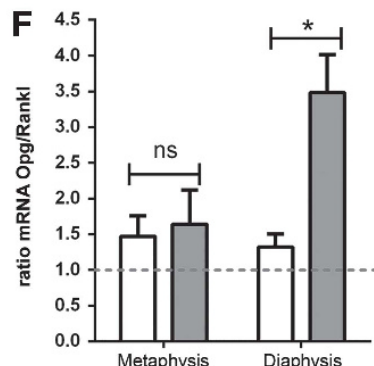

Figure 4 Effects of DlX3 deletion on osteoclastic bone resorption in 5-week-old male mice. (A) Serum levels of CTX-1 were not significantly affected in DIX3 ${ }^{O C N-c K O}$ mice $(n=5)$ when compared with D/x $3^{+/+}$mice $(n=3)$. Bottom panel, TRAP staining was performed on femur sections and computerized images of trabecular bone were used for histomorphometric analysis. TRAP-positive cells (TRAP $(+)$ cells) and TRAP-positive surface (TRAP $(+)$ S) in metaphysis and diaphysis areas were normalized against the matrix bone surface (BS). Both parameters showed no significant difference between $D / x 3^{O C N}$-cKO mice $(n=2)$ when compared with $D / x 3^{+1+}$ littermates $(n=2)$. (B) Osteoclasts were visualized using TRAP staining on sections from decalcified and paraffin-embedded 5 wk $D / x 3^{+/+}$and $D / x 3^{O C N-c K O}$ femurs. Metaphysis (a, b) and diaphysis (c, d) are shown in higher magnification (inserts). (C, D) M-BMMs (M-CSF-dependent bone marrow macrophages) were isolated from femur and tibia from $D / x 3^{O C N-C K O}$ and $D / x 3^{+1+} 5$ wk males and cultured in presence of $50 \mathrm{ng} / \mathrm{ml} \mathrm{M-CSF}$ and various concentration of RANKL $(0,10,30,50 \mathrm{ng} / \mathrm{ml})$. (C) TRAP activity staining was performed at D6. (D) mRNA expression of Trap was monitored at D6 by Q-PCR. mRNA levels have been normalized to the expression levels of the housekeeping gene

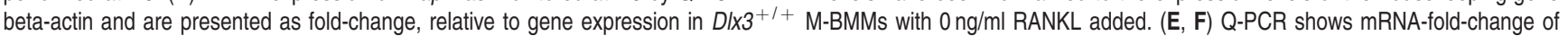

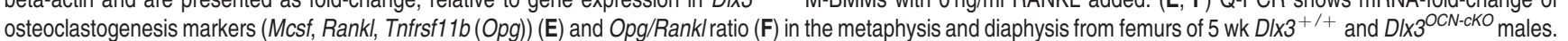
Data are presented as the mean \pm S.E.M. ns: non-significant, ${ }^{*} P<0.05$. Scale bars: $500 \mu \mathrm{m}$ for the main figures (letters) and $100 \mu \mathrm{m}$ for the magnification boxes

\section{DLX3 alters osteoblast differentiation by modulating the binding of bone-activator TFs on regulatory elements} on the OCN promoter. The Ocn gene is repressed in proliferating osteoprogenitor cells and highly induced in mature osteoblasts, producing a mineralized matrix. DLX3 is primarily bound to the Ocn promoter at the onset of osteoblastogenesis, whereas DLX5 associates with Ocn during mineralized tissue formation. ${ }^{9}$ We examined by ChIP the proximal Ocn promoter containing the OC box (Figure 7), and observed that DLX3-deficient calvarial osteoblasts had a significant increase in the amount of RUNX2 occupying the Ocn promoter in both the proliferative stage (Day 4,5$)$ as well as matrix maturation stages (Day 9, 12, 18) compared with Adv-GFP cells (Figure 7b). Because other homeobox proteins such as DLX5 have been demonstrated to bind to the same regulatory region and regulate Ocn gene expression, ${ }^{9}$ we investigated if the promoter occupancy of DLX5 was altered in cells lacking DLX3. In proliferating
(Day 4, 5) DLX3-deficient osteoprogenitors, DLX5 occupancy of the Ocn proximal promoter was significantly increased (Figure 7b) but did not show an appreciable difference during the differentiation/matrix maturation stages (Day 9-18) that corresponds to an exponential increase in Ocn mRNA transcription. Taken together, these data suggest that Ad-Cre excision of D/x3 in calvarial osteoblasts resulted in increased expression of osteoblast-related markers and an increased occupancy of DLX5 at the Ocn homeodomain element as well as the increased and earlier occupancy of RUNX2.

\section{Discussion}

Herein we demonstrate a distinct role for DLX3 as an essential regulator of three integral processes of EBF and maintenance: osteoblastogenesis, matrix deposition and bone homeostasis. In DIx $3^{c K O}$ mice, the coordinated process of 
a

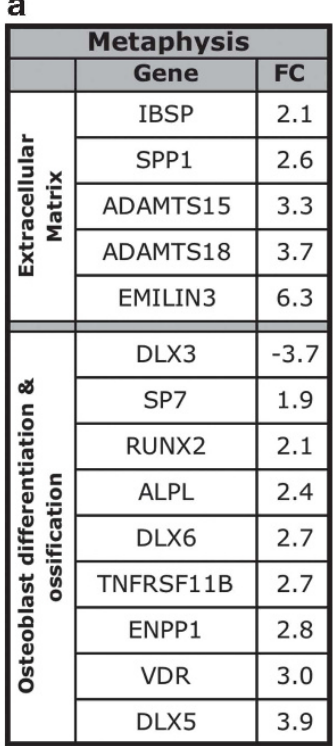

b

\begin{tabular}{|c|c|c|}
\hline \multicolumn{3}{|c|}{ Diaphysis } \\
\hline & Gene & FC \\
\hline \multirow{4}{*}{ 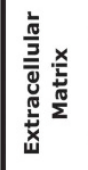 } & ADAMTS15 & 2.0 \\
\hline & MEPE & 2.4 \\
\hline & EMILIN3 & 3.0 \\
\hline & ADAMTS18 & 3.2 \\
\hline \multirow{4}{*}{ 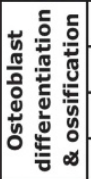 } & DLX3 & -7.2 \\
\hline & ENPP1 & 1.9 \\
\hline & TNFRSF11B & 1.9 \\
\hline & VDR & 2.2 \\
\hline
\end{tabular}

c
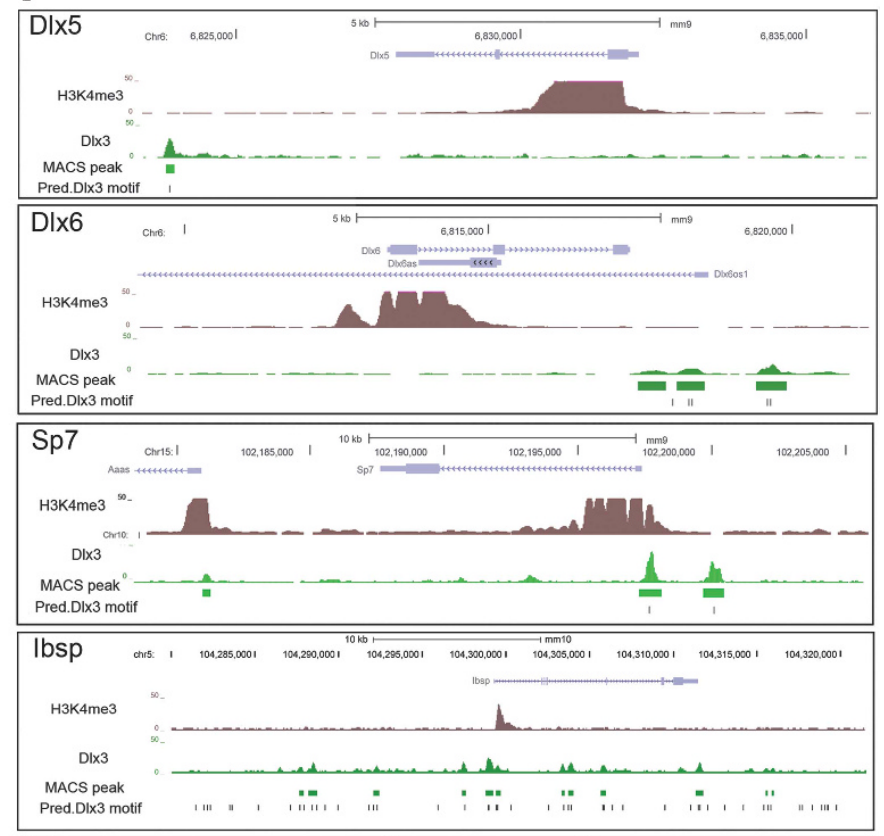

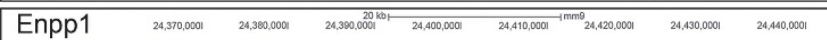

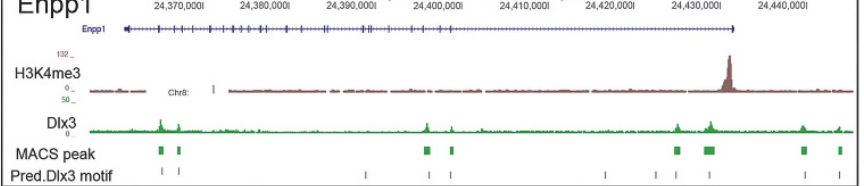

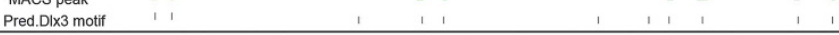
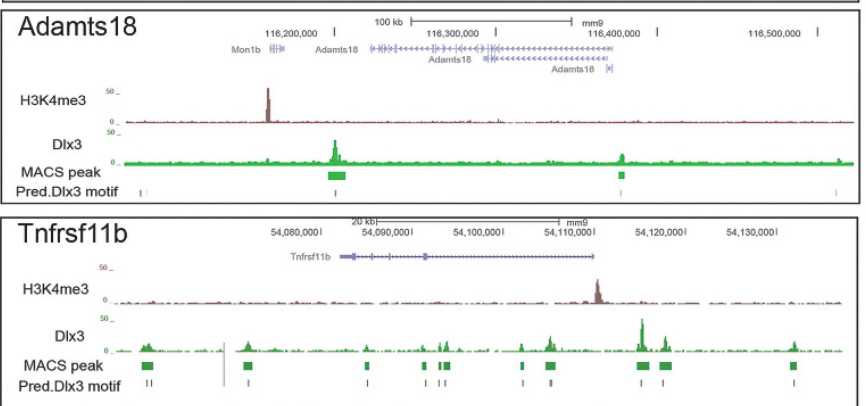

Figure 5 RNA-Seq and ChIP-Seq on DLX3-deficient bones and osteoblasts identify molecular targets of Dlx3 regulation of bone mass. RNA-Seq differential gene expression profiling was performed on femurs from $5 \mathrm{wk} D / \times 3^{+/+}$and $D / \times 3^{O C N-c K O}$ males. Selected genes differentially expressed in the metaphysis (a) and diaphysis (b) of $D / x 3^{\mathrm{OCN}-\mathrm{cKO}}$ compared with $\mathrm{D} / \times 3^{+/+}$mice genes are organized by genes involved in ECM and genes related to the osteoblastic differentiation and ossification processes. (c) ChIP-Seq analysis performed in SMAA-positive BMSCs shows DLX3 binding to bone-related genes differentially regulated in D/x $3^{\mathrm{OCN}-\mathrm{CKO}}$ mice identified by RNA-Seq. Tracks for DIx5, DIx6, Sp7, Ibsp, Enpp1, Adamts18 and Tnfrsf11b (Opg) were visualized on UCSC genome browser with Refseq displayed for gene annotation and H3K4me3 track provided as reference

EBF at the growth plate was retained, although the amount of bone tissue deposited was significantly enhanced. Trabecular bone extended into the medullary cavity; ALPL staining was enhanced, and bone volume ratio and trabecula number was increased. Cortical bone was also notably thicker with an increased MAR but also featured increased porosity and decreased BMD.

We previously reported the function of DLX3 in intramembranous bones of neural crest origin in the craniofacial area. Similar to the present study, Wnt1cre:DIx3 mice exhibited decreased BMD in mandibular and calvarial bones and increased porosity in mandible. ${ }^{12}$ Furthermore, here we show that adenoviral-Cre-infected calvarial cells and longbone BMSCs isolated from $D / \times 3^{F / F}$ neonates and 5 wk mice, respectively, showed increased differentiation capacity as demonstrated by ALPL staining, which is consistent with calvarial cell differentiation from Wnt1cre:DIx3 neonates. ${ }^{12}$ However, craniofacial bones from Wnt1cre:Dlx3 neonates and femurs from $D / \times 3^{O C N-C K O} 5 \mathrm{wk}$ mice had a particular gene signature where few genes including Alpl, Ibsp, Mepe, Ihh and Adamst18 were commonly affected. We presently show that although expression of Runx2 and DIx5 were significantly upregulated in DIx3-deleted BMSCs from long bones of $5 \mathrm{wk}$ $D / x 3^{F / F}$, these genes were not affected in Dlx3-deleted calvarial cells isolated from $D / x 3^{F / F}$ neonates. This indicates that $D / x 3$ regulates osteoblast differentiation in intramembranous and endochondral bones via tissue- or stage-specific molecular mechanisms. This hypothesis is further supported 
a

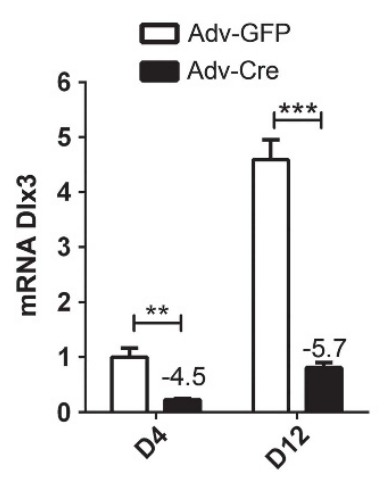

b

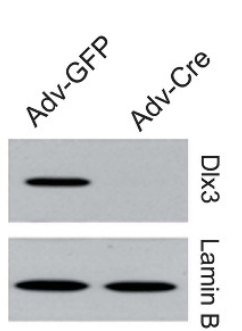

c
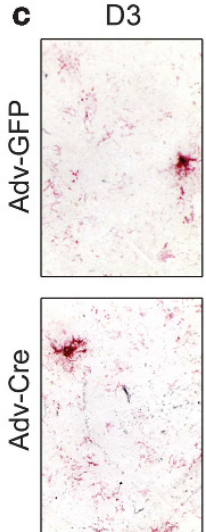

D5

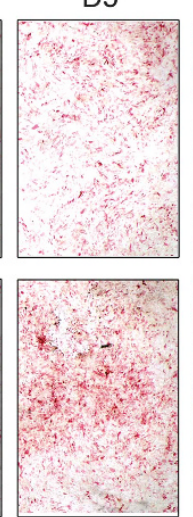

D7
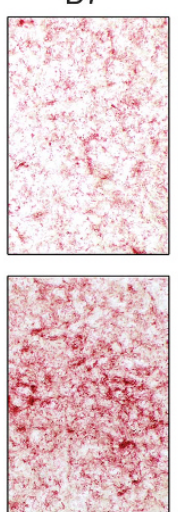

D12

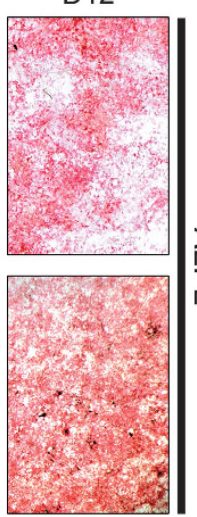

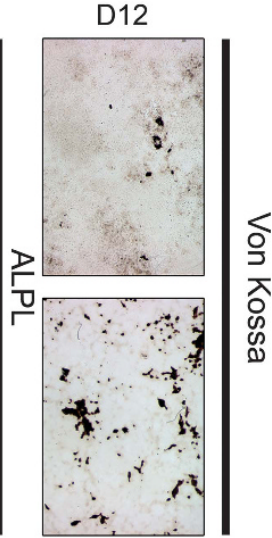
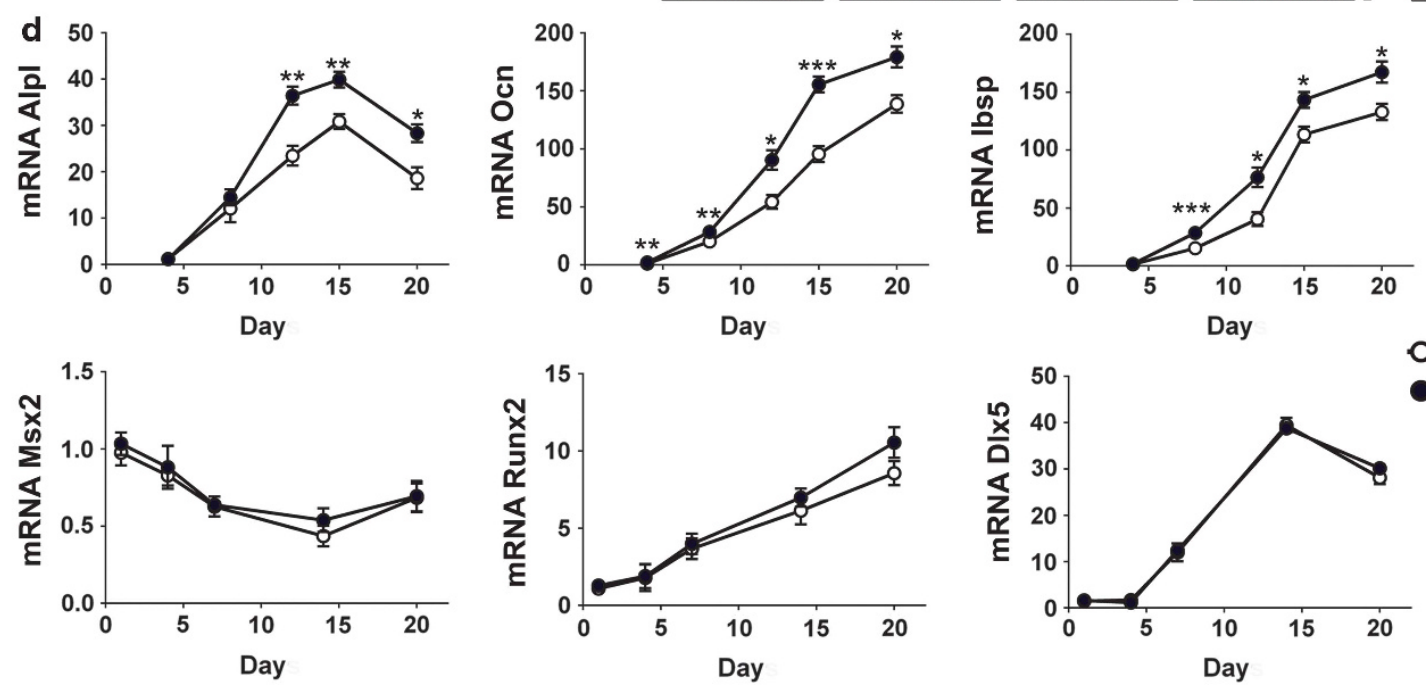

O Adv-GFP

- Adv-Cre

Figure 6 D/x3 excision in calvarial osteoblasts increases expression of osteoblast markers. (a) Q-PCR of DIx3 mRNA expression represented as fold-change in Adv-GFP and Adv-Cre-infected calvarial osteoblasts from D/x3 $3^{F / F}$ neonates during proliferating (D4) and matrix maturation stages (D12). (b) Western blot of DLX3 and Lamin B in virus-infected calvarial osteoblasts at D12. (c) ALPL and Von Kossa stainings of virus-infected calvarial osteoblasts demonstrated increased ALPL activity and mineralization capacity in Adv-Cre-infected cells. (d) Q-PCR of mRNA expression represented as fold-change of osteoblast-related markers (Alpl, Ibsp, Ocn) and bone transcription factors (Runx2, Msx2, D(x5). Data are presented as the mean \pm S.E.M. ${ }^{\star} P<0.05,{ }^{\star \star} P<0.01,{ }^{\star \star \star} P<0.001$

by our transcriptome studies which reveal that in vivo DIX3 deletion in osteogenic cells affects a wide range of developmental signals including Wnt, Tgf- $\beta / \mathrm{Bmp}$ and Notch pathways, but only few genes encoding signaling molecules are commonly affected in the Wnt1cre:DIx3 and DIx $3^{O C N-c K O}$ models.

Appendicular skeleton and craniofacial bones display differential molecular fingerprints, ${ }^{26}$ one notable difference being that in the appendicular skeleton skeletal patterning and osteoblastogenesis are regulated by $\mathrm{HOX}$ TFs, ${ }^{20}$ whereas most of the craniofacial bones are Hox free. This Hox status would be determinant in osteogenic potential and cellular plasticity. ${ }^{27}$ In the present study, $D / x 3$ deletion resulted in upregulation of $H o x A 3 / B 2 / B 3 / B 4$. Our results support that the transcriptional network required for proper EBF involves a direct or indirect inhibition by DLX3 of the Hox gene family and their downstream Hox-specific gene regulatory network. In line with this hypothesis, Hassan et al. ${ }^{28}$ previously demonstrated the selective association of HOXA10 and DLX3 to the Runx2 and Ocn regulatory promoter regions, an association correlated with the stages of osteoblast maturation. We hypothesize that this difference in HOX regulation, along with aforementioned differentially regulated TFs and osteoblastogenic markers contribute to the tissuespecific differences in DLX3-dependent network regulation between intramembranous and endochondral bones.

In the TDO model, transgenic mice lacked an enhanced dynamic bone formation rate and the increased bone volume and BMD trabecular phenotype was attributed to decreased osteoclast bone resorption activity due to increased IFN- $\gamma{ }^{13}$ In contrast, our analyses in $D / \times 3^{O C N-C K O}$ mice support that increased trabecular bone mass does not arise from impaired osteoclastic activity but from direct enhancement of the boneforming osteoblast activity. This leads to accelerated bone formation, thereby inducing an imbalance in homeostasis in favor of bone apposition.

In support of increased osteoblast activity due to $D / x 3$ deletion, RNA-Seq demonstrated that in DIx $3^{O C N-c K O}$ metaphysis, positive TFs of osteoblastogenesis were upregulated, including Runx $2^{29}$ and its downstream osteoblastspecific target $S p 7 .^{30} D / \times 5$ and D/x6, two biologically equivalent positive regulators of chondrocyte and osteoblast 
a
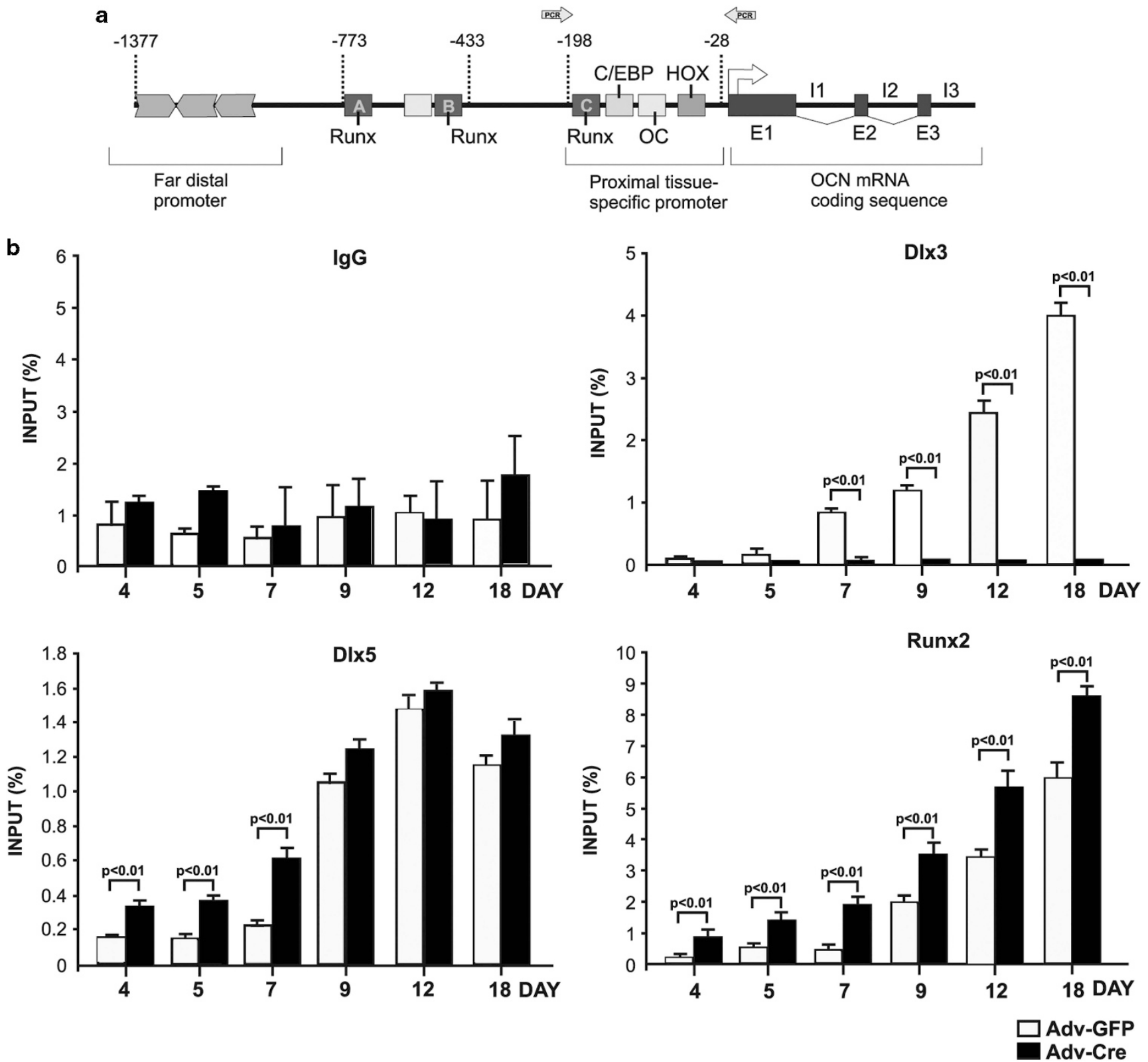

Figure 7 Increased occupancy of RUNX2 on the osteocalcin promoter in DLX3-deficient calvarial osteoblasts. (a) Diagram of the mouse osteocalcin promoter displaying relative binding sites and primer sites used for chromatin immunoprecipitation analysis. (b) Calvarial osteoblasts isolated from $D / \times 3^{\mathrm{F} / F}$ mice were infected with $\mathrm{Adv}$-Cre or Adv-GFP and cultured in osteogenic media for 18 days. ChIP was then preformed on cleared cell lysates on the indicated day using $\sim 5 \mu \mathrm{g}$ of RUNX2, DLX3, DLX5, or non-specific $\lg G$ antibody. Recovered DNA was then quantified by $Q-P C R$ and normalized to input. Data are presented as the mean of three experiments \pm S.E.M. ${ }^{*} P<0.05$, ${ }^{\star \star} P<0.01,{ }^{\star \star *} P<0.001$

differentiation ${ }^{6,31-34}$ were also upregulated in $D / x 3^{O C N-c K O}$ metaphysis. Given that all four networked genes have been characterized as stimulators of osteoblast differentiation and activity, we hypothesize that increased DIx5,DIx6, Runx2 and $S p 7$ expression in $D / \times 3^{O C N-c K O}$ mice contributes to the significantly increased trabecular bone volume and number of trabeculae. This hypothesis is further supported by our in vitro model; DIx3-deleted BMSCs showed an increased osteoblast differentiation and bone-forming activity associated with increased gene expression of Runx2 and DIx5. Both our in vivo and in vitro data, together with previous studies reporting in vivo loss of $D / x 3$ expression in
$D / x 5 / D / x 6^{-1-}$ mutants $^{35}$ suggest a mutually negative regulatory loop between DLX5/DLX6 and DLX3 during bone formation and homeostasis. ChIP analysis on BMSCs demonstrated that DLX3 binds to the promoters of Sp7, DIx5 and DIx6. These data together with previous in vitro studies showing that DLX3 can bind to the Runx2 promoter and directly modulate its transcription ${ }^{10}$ lead us to hypothesize that in long bones, DLX3 could attenuate osteoblastogenesis by reducing Runx2, Sp7, DIx5 and DIx6 gene expression.

Upregulation of several bone ECM genes, including Ibsp and Spp1 and local regulators of bone mineralization such as Alpl and Enpp1 was also observed in DIx $3^{O C N-c K O}$ 
metaphysis. ChIP analysis of long-bone cells demonstrated potential DLX3 binding to Enpp1 and Ibsp promoters. Enpp1 is necessary for osteoblast differentiation and inhibits bone

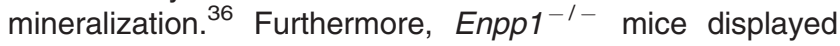
trabecular bone loss and hyper-mineralization in long bones. ${ }^{17} \mathrm{lbsp} \mathrm{fl}^{-1-}$ mice displayed impaired bone growth and mineralization and dramatically reduced bone formation. ${ }^{37}$ We postulate that increased Enpp1 and Ibsp expression in $D / x 3^{O C N-C K O}$ metaphysis could contribute to the increased trabeculae phenotype. Collectively these data support that DLX3 regulation of ECM genes and major regulators of bone mineralization is necessary for conventional matrix formation.

Different stages of osteoblast differentiation - proliferation, maturation and mineralization - are governed by coordinated TFs binding on promoters of bone-related genes to regulate their temporal expression. ${ }^{28}$ In vitro studies have shown that DLX3 and DLX5 associate with the Ocn promoter at the onset of transcriptional activation, concomitant with RUNX2 occupancy, and whereas DLX3 occupancy on the Ocn promoter decreased from osteoblast maturation to mineralization, DLX5 occupancy was maximal during the mineralization stage. ${ }^{9}$ In the present study, we demonstrated that $D / \times 3$ deletion in calvarial osteoblasts results in increased occupancy of DLX5 and increased and premature occupancy of RUNX2 on regulatory elements on the Ocn promoter. These data suggest that $D / x 3$ deletion could directly affect the network of TF molecular switches and promote osteoblastic differentiation and bone-forming activity via an enhancement of the occupancy of bone-activator TFs such as DLX5 and RUNX2. Among the genes differentially expressed in $D / \times 3^{O C N-C K O}$ metaphysis, DLX5 has been shown to regulate and bind to the promoter regions of Runx $2,{ }^{10}$ Hox family members, ${ }^{28} S p 7,{ }^{38} A / p{ }^{39}$ and $l b s p .{ }^{40,41}$ These data together with our ChIP results demonstrating DLX3 binding to some of these genes lead us to speculate that DLX3 and DLX5 may have a coordinated role in the transcription of bone-related genes via molecular switches at their promoter regions.

We also performed in-depth characterization of the $D / \times 3^{O C N-C K O}$ cortical bone and found increased thickness associated with increased periosteum MAR, decreased BMD and increased porosity; also, these porous regions were vascularized. It remains to be determined whether this increased vascularization and decreased BMD result from the high bone mass phenotype of $D / x 3^{c K O}$ mice or from the upregulation of modulators of bone mineralization and porosity such as Enpp 1. ${ }^{17}$ In cortical bone, RNA-Seq showed upregulation of Opg and Mepe, two major inhibitors of osteoclastogenesis strongly expressed by late-differentiated osteoblasts and osteocytes. ${ }^{21,42-44}$ ChIP analysis revealed that DLX3 was bound to multiple regions of Opg. This supports a model in which D/x3 deletion in osteoblasts induces increased levels of Opg and Mepe in cortical bone, and thereby deregulates normal homeostasis in favor of a positive bone balance. Samee et al. ${ }^{33}$ reported that $D / x 5^{-1-}$ mice had an increased number of osteoclasts and $D / x 5^{-1-}$ osteoblasts exhibited decreased Opg expression resulting in a higher Rankl/Opg ratio. This suggests that, as previously shown for DLX5, ${ }^{33} \mathrm{DLX} 3$ is a central regulator of osteoblast/ osteoclast coupling. However, conversely to DLX5, DLX3 would inhibit osteoblast bone-forming activity via a direct or indirect negative transcriptional control of genes responsible for bone formation while simultaneously activating bone resorption through regulation of osteoblastic modulators of osteoclastogenesis.

Collectively, our studies establish that DLX3 has an important role in regulating endochondral bone mass throughout the lifespan. We propose that DLX3 acts as a negative modulator that regulates expression of crucial bone-related genes throughout osteoblastogenesis, bone matrix synthesis and skeleton homeostasis. These results suggest that DLX3 is an attractive translational target for stimulating bone formation in skeletal disorders.

\section{Materials and Methods}

Mice breeding. Cre recombinase activity was traced by mating Prx1-cre and $\mathrm{OCN}$-cre mice with Rosa LacZ or YFP mice. For details, see Supplementary information. All animal work was approved by the NIAMS Animal Care and Use Committee.

Histological and immunohistochemical (IHC) analyses. Samples were fixed, dehydrated, decalcified, embedded and cut in $10-\mu \mathrm{m}$ sections. Toluidine blue stained for bone cartilage. ALPL (R\&D Systems, Minneapolis, MN, USA) antibody was used 1:200 with secondary anti-rat antibody 1:400 (Vector Laboratories, Burlingame, CA, USA). For detailed IHC methods, see Supplementary information.

Bone tissue collection and RNA extraction. Long bones were harvested at P3.5, P9.5 and 5 wk. In 5-wk-male mice, cartilage caps from femur were removed and the metaphysis was harvested. Bone marrow cells were flushed with $1 \times$ PBS using a 27-gauge needle. The remaining cortical bone was harvested as the diaphysis. For RNA extraction details, see Supplementary information.

Q-PCR. cDNA was prepared from RNA and Q-PCR analysis was performed for target genes, run in triplicate for each sample. Gene expression levels were normalized to the expression of beta-actin and presented as FC, relative to WT expression. Oligonucleotides used are summarized in Supplementary Table S1 and detailed Q-PCR methods are in Supplementary information.

Western blot. Primary antibodies used were anti-DLX3 $(1: 2000)$ and antiGAPDH (1:5000; Abcam, Cambridge, MA, USA). Secondary antibodies used were goat anti-rabbit HRP for DLX3 $(1: 3000)$ and goat anti-mouse HRP for GAPDH $(1: 5000)$ (Bio-Rad, Hercules, CA, USA).

$\mu \mathbf{C T}$ analysis. Fixed femurs and tibias from 2 wk to $6 \mathrm{mo} D / \times 3^{+/+}$, $D / x 3^{O C N-C K O}$ and $D / x 3^{P r \times 1-C K O}$ mice $(n=2$ mice minimum per group) were scanned at $10 \mu \mathrm{m}$ voxel resolution ( $\mu \mathrm{CT} 40$; Scanco Medical AG, Brutisellen, Switzerland) and images were reconstructed using Scanco software v5.0. Trabecular and cortical bone areas were selected and analyzed as previously described. ${ }^{45}$ Parameters were obtained using thresholds range 220-1000 and a density range superior to $500 \mathrm{mg}$ of $\mathrm{HA} / \mathrm{cm}^{3}$.

Fluorochrome measurement of bone formation rates. Calcein labeling was performed on 5 wk mice as previously described. ${ }^{45}$ Distances between two calcein labels were measured at 4-6 points along the periosteal surface of the cortical bone in $n=2 D / \times 3^{+/+}$and D/x $3^{\mathrm{OCN}-\mathrm{CKO}}$ mice. The points were added together and the mean \pm S.E.M. was plotted for each mouse.

RNA-Seq. mRNA isolated from 5 wk metaphysis and diaphysis was processed with Illumina TruSeq RNA Sample Preparation kit (Illumina, San Diego, CA, USA), and data were generated with an Illumina HiSeq 2000 sequencing system. Criteria for significant gene selection included $q$-values $\leq 0.05$ (for multi-test adjustment), $\mathrm{FC} \geq 1.85$ or $\leq-1.85$, and mean $\mathrm{RPKM} \geq 1$ for the mutant group in selecting upregulated genes, or for the control group in selecting downregulated genes. For detailed RNA-Seq methods, see Supplementary information. 
Measurement of serum CTX-1 level. The CTX-1 Ratlaps ELISA was performed with serum from 5 wk $D / \times 3^{+/+}$and $D / \times 3^{O C N-C K O}$ mice according to manufacturer protocol (Immunodiagnostic Systems Inc., Scottsdale, AZ, USA).

TRAP activity assay. A TRAP activity assay was performed as previously described. ${ }^{46}$ For detailed quantification methods, see Supplementary information.

M-CSF-dependent bone marrow macrophages (M-BMM) isolation and culture. For osteoclast experiments, BMSCs isolated from femurs and tibias of 5 wk D/x $3^{+1+}$ and D/x3 ${ }^{\mathrm{OCN}-\mathrm{CKO}}$ males were treated with $50 \mathrm{ng} / \mathrm{ml}$ of MCSF (R\&D Systems) and various concentrations $(0,10,30$ and $50 \mathrm{ng} / \mathrm{ml}$ ) of RANKL (R\&D Systems). For BMSCs and M-BMMs culturing and TRAP staining methods, see Supplementary information.

ChIP and high-throughput sequencing of DLX3-associated DNA in BMSCs. For ChIP-Seq studies (Figure 5), BMSCs were isolated from femurs and tibias of 6 -wk-mice expressing mCherry aSMA promoter. ${ }^{47}$ Cells were initially FACS sorted to enrich for a homogenous population of progenitor cells for osteoblast differentiation. $1 \times 10^{8}$ BMSCs were washed with PBS and then fixed with $1 \%$ formaldehyde for 10 min to crosslink DNA-protein complexes. Isolated chromatin was obtained as previously described ${ }^{9}$ and used for immunoprecipitation with anti-DLX3 antibody (ab66390, Abcam) or IgG as a control followed by Protein-G Dynabeads (Invitrogen, Carlsbad, CA, USA). DNA was amplified using the Illumina system (Illumina) and sequenced on an Illumina Genome Analyzer II. For BMSCs isolation and culturing methods and detailed ChIP data analysis, see Supplementary information.

Calvarial osteoblast culture, excision of D/x3 allele by adenoviral-Cre recombinase infection. Primary mouse calvarial osteoblasts were isolated from neonates containing two floxed $D / \times 3$ alleles $\left(D / \times 3^{F / F}\right)$ using sequential collagenase. ${ }^{9}$ Isolated cells were infected with Cre recombinase or GFP-containing Adv (University of lowa Gene Transfer Vector Core) at an m.o.i. of 100 for $6 \mathrm{~h}$. Cells were assessed for D/x3 excision by Q-PCR. For osteogenic differentiation, aMEM was further supplemented with $280 \mu \mathrm{M}$ ascorbic acid and $10 \mathrm{mM}$ b-glycerophosphate. Whole-cell lysates from Adv-Cre or Adv-GFP infected primary calvarial cells $\left(\sim 1 \times 10^{7}\right.$ cells $)$ were analyzed via western blot as previously described. ${ }^{9}$ Primary antibodies anti-DLX3 (C-20, Santa Cruz Biotechnologies, Santa Cruz, CA, USA) or anti-Lamin B1 (1:1000; Invitrogen) were used. Secondary antibodies were species-specific HRP-conjugated antibodies (1: 10000 ; Santa Cruz Biotechnologies). ALPL activity and Von Kossa staining assay were performed as previously described. ${ }^{12}$ For Q-PCR, cDNA was made and levels were normalized to the expression of beta-actin represented as FC, relative to target gene expression in Adv-GFP calvarial osteoblasts at D4.

ChIP assay in DLX3-deficient calvarial osteoblasts. ChIP assays were performed as previously described. ${ }^{9}$ Briefly, calvarial osteoblasts infected with Cre recombinase or GFP-containing Adv were collected from $D / \times 3^{F / F}$ neonates, crosslinked, lysed and genomic DNA sheared by sonication. Diluted lysates were then immunoprecipitated with protein-specific antibodies; anti-RUNX2 (PEBP2aa) (M-70; sc-10758), anti-DLX3 (C-20; sc-18143), anti-DLX5 (C-20; sc-18152) or IgG as a non-specific binding control (Santa Cruz Biotechnologies). Aliquots of ChIP samples were assayed by Q-PCR to detect the presence of specific DNA fragments using oligos from the mouse OCN (Ocn also known as Bglap2) proximal promoter spanning bp -198 to -28 ; forward, $5^{\prime}$-GGC AGC CTC TGA TTG TGT CC-3'; reverse, 5'-TAT ATC CAC TGC CTG AGC GG-3'. For each immunoprecipitation, DNA levels were normalized to input. For calvarial cell isolation, culturing and infection, see Supplementary information.

Statistical analyses. FC is defined as $\left(n^{\text {mutant }} / n^{W T}\right)$ if this ratio is $\geq 1$ or $-\left(\mathrm{n}^{\mathrm{WT}} / \mathrm{n}^{\text {mutant }}\right)$ if the ratio is $<1$. Statistical analyses of the Q-PCR data, CTX-1 data and TRAP-related parameters were performed on Prism 5 software (GraphPad Software, La Jolla, CA, USA), using the Mann-Whitney test. $\mu \mathrm{CT}$ data were analyzed with Partek GS 6.6 using two-way ANOVA. A random 'litter' factor is included to account for the litter effect. For all tests, the significant $P$-value limit is 0.05 . All quantitative experiments were performed on at least two control and two mutant animals and for cell culture, at least two independent experiments were run. Data are expressed as mean \pm S.E.M. Statistical analyses for RNA-Seq and ChIP data are described in related sections.

\section{Conflict of Interest}

The authors declare no conflict of interest.

Acknowledgements. We thank Dr. T Clemens for the OCN-cre mice; S Russell, of the University of Massachusetts MusculoSkeletal Imaging Facility for performing $\mu \mathrm{CT}$ analyses; Dr. K Zaal of the NIAMS Light Imaging Core Facility; G Gutierrez-Cruz of the Genome Analyzer Core Facility; Drs. A Maeda and $M$ Young of the NIDCR, NIH; Dr. B Foster of NIAMS, NIH for assistance with ALPL staining, Audrey Asselin of the INSERM UMRS 1138 (Team Berdal), for assistance with cell culture, and Christophe Klein of the INSERM UMRS 1138 (CICC) and Laura Solomon (UVM graduate student) for osteoclast quantification. The Morasso and Lian laboratories and their funding contributed equally to this project. This research was supported by the Intramural Research Program of the National Institute of Arthritis and Musculoskeletal and Skin Diseases of the NIH. JBL is supported by NIH grant R37 DE012528.

1. Cohen MM Jr. Perspectives on RUNX genes: an update. Am J Med Genet A 2009; 149A: 2629-2646.

2. Jensen ED, Gopalakrishnan R, Westendorf JJ. Regulation of gene expression in osteoblasts. Biofactors 2010; 36: 25-32.

3. Long F. Building strong bones: molecular regulation of the osteoblast lineage. Nat Rev $\mathrm{Mol}$ Cell Biol 2012; 13: 27-38.

4. Depew MJ, Simpson CA, Morasso M, Rubenstein JL. Reassessing the Dlx code: the genetic regulation of branchial arch skeletal pattern and development. J Anat 2005; 207: 501-561.

5. Panganiban G, Rubenstein JL. Developmental functions of the Distal-less/Dlx homeobox genes. Development 2002; 129: 4371-4386.

6. Samee N, de Vernejoul MC, Levi G. Role of DLX regulatory proteins in osteogenesis and chondrogenesis. Crit Rev Eukaryot Gene Expr 2007; 17: 173-186.

7. Shamseldin HE, Faden MA, Alashram W, Alkuraya FS. Identification of a novel DLX5 mutation in a family with autosomal recessive split hand and foot malformation. $J$ Med Genet 2012; 49: 16-20.

8. Haldeman RJ, Cooper LF, Hart TC, Phillips C, Boyd C, Lester GE et al. Increased bone density associated with DLX3 mutation in the tricho-dento-osseous syndrome. Bone 2004; 35: 988-997.

9. Hassan MQ, Javed A, Morasso MI, Karlin J, Montecino M, van Wijnen AJ et al. Dlx3 transcriptional regulation of osteoblast differentiation: temporal recruitment of Msx2, DIx3, and Dlx5 homeodomain proteins to chromatin of the osteocalcin gene. Mol Cell Biol 2004; 24: 9248-9261.

10. Hassan MQ, Tare RS, Lee SH, Mandeville M, Morasso MI, Javed A et al. BMP2 commitment to the osteogenic lineage involves activation of Runx2 by DLX3 and a homeodomain transcriptional network. J Biol Chem 2006; 281: 40515-40526.

11. Singh M, Del Carpio-Cano FE, Monroy MA, Popoff SN, Safadi FF. Homeodomain transcription factors regulate BMP-2-induced osteoactivin transcription in osteoblasts. $J$ Cell Physiol 2012; 227: 390-399.

12. Duverger O, Isaac J, Zah A, Hwang J, Berdal A, Lian JB et al. In vivo impact of Dlx3 conditional inactivation in neural crest-derived craniofacial bones. J Cell Physiol 2013; 228: 654-664.

13. Choi SJ, Roodman GD, Feng JQ, Song IS, Amin K, Hart PS et al. In vivo impact of a 4 bp deletion mutation in the DLX3 gene on bone development. Dev Biol 2009; 325 : 129-137.

14. Lezot F, Thomas BL, Blin-Wakkach C, Castaneda B, Bolanos A, Hotton D et al. Dlx homeobox gene family expression in osteoclasts. J Cell Physiol 2010; 223: 779-787.

15. Doi M, Nagano A, Nakamura Y. Molecular cloning and characterization of a novel gene, EMILIN-5, and its possible involvement in skeletal development. Biochem Biophys Res Commun 2004; 313: 888-893.

16. Li YC, Pirro AE, Amling M, Delling G, Baron R, Bronson R et al. Targeted ablation of the vitamin $\mathrm{D}$ receptor: an animal model of vitamin D-dependent rickets type II with alopecia. Proc Natl Acad Sci USA 1997; 94: 9831-9835.

17. Mackenzie NC, Zhu D, Milne EM, van 't Hof R, Martin A, Darryl Quarles $L$ et al. Altered bone development and an increase in FGF-23 expression in Enpp1 $(-/-)$ mice. PLoS One 2012; 7: e32177.

18. Narisawa S, Frohlander N, Millan JL. Inactivation of two mouse alkaline phosphatase genes and establishment of a model of infantile hypophosphatasia. Dev Dyn 1997; 208: 432-446.

19. Staines KA, MacRae VE, Farquharson C. The importance of the SIBLING family of proteins on skeletal mineralisation and bone remodelling. J Endocrinol 2012; 214: 241-255.

20. Wellik DM. Hox genes and vertebrate axial pattern. Curr Top Dev Biol 2009; 88: 257-278.

21. Petersen DN, Tkalcevic GT, Mansolf AL, Rivera-Gonzalez R, Brown TA. Identification of osteoblast/osteocyte factor 45 (OF45), a bone-specific cDNA encoding an RGD-containing protein that is highly expressed in osteoblasts and osteocytes. J Biol Chem 2000; 275: 36172-36180.

22. Chen G, Deng C, Li YP. TGF-beta and BMP signaling in osteoblast differentiation and bone formation. Int J Biol Sci 2012; 8: 272-288. 
23. Gazzerro E, Canalis E. Bone morphogenetic proteins and their antagonists. Rev Endocr Metab Disord 2006; 7: 51-65.

24. Monroe DG, McGee-Lawrence ME, Oursler MJ, Westendorf JJ. Update on Wnt signaling in bone cell biology and bone disease. Gene 2012; 492: 1-18.

25. Zanotti S, Canalis E. Notch signaling in skeletal health and disease. Eur J Endocrinol 2013; 168: R95-103

26. Rawlinson SC, McKay IJ, Ghuman M, Wellmann C, Ryan P, Prajaneh S et al. Adult rat bones maintain distinct regionalized expression of markers associated with their development. PLoS One 2009; 4: e8358.

27. Leucht P, Kim JB, Amasha R, James AW, Girod S, Helms JA. Embryonic origin and Hox status determine progenitor cell fate during adult bone regeneration. Development 2008; 135: $2845-2854$

28. Hassan MQ, Saini S, Gordon JA, van Wijnen AJ, Montecino M, Stein JL et al. Molecular switches involving homeodomain proteins, HOXA10 and RUNX2 regulate osteoblastogenesis. Cells Tissues Organs 2009; 189: 122-125.

29. Otto F, Thornell AP, Crompton T, Denzel A, Gilmour KC, Rosewell IR et al. Cbfa1, a candidate gene for cleidocranial dysplasia syndrome, is essential for osteoblast differentiation and bone development. Cell 1997; 89: 765-771.

30. Nakashima K, Zhou X, Kunkel G, Zhang ZP, Deng JM, Behringer RR et al. The novel zinc finger-containing transcription factor Osterix is required for osteoblast differentiation and bone formation. Cell 2002; 108: 17-29.

31. Ferrari D, Sumoy L, Gannon J, Sun HL, Brown AMC, Upholt WB et al. The expression pattern of the distal-less homeobox-containing gene Dlx-5 in the developing chick limb bud suggests its involvement in apical ectodermal ridge activity, pattern-formation, and cartilage differentiation. Mech Dev 1995; 52: 257-264.

32. Lee MH, Kim YJ, Yoon WJ, Kim JI, Kim BG, Hwang YS et al. Dlx5 specifically regulates Runx2 type II expression by binding to homeodomain-response elements in the Runx2 distal promoter. J Biol Chem 2005; 280: 35579-35587.

33. Samee N, Geoffroy V, Marty C, Schiltz C, Vieux-Rochas M, Levi G et al. Dlx5, a positive regulator of osteoblastogenesis, is essential for osteoblast-osteoclast coupling. $A m \mathrm{~J}$ Pathol 2008; 173: 773-780.

34. Tadic T, Dodig M, Erceg I, Marijanovic I, Mina M, Kalajzic Z et al. Overexpression of Dlx5 in chicken calvarial cells accelerates osteoblastic differentiation. J Bone Miner Res 2002; 17 1008-1014.

35. Depew MJ, Lufkin T, Rubenstein JL. Specification of jaw subdivisions by Dlx genes. Science 2002; 298: 381-385.

36. Nam HK, Liu J, Li Y, Kragor A, Hatch NE. Ectonucleotide pyrophosphatase/ phosphodiesterase-1 (ENPP1) protein regulates osteoblast differentiation. $\mathrm{J}$ Biol Chem 2011; 286: 39059-39071.
37. Malaval L, Wade-Gueye NM, Boudiffa M, Fei J, Zirngibl R, Chen F et al. Bone sialoprotein plays a functional role in bone formation and osteoclastogenesis. J Exp Med 2008; 205: 1145-1153.

38. Ulsamer A, Ortuno MJ, Ruiz S, Susperregui AR, Osses N, Rosa JL et al. BMP-2 induces Osterix expression through up-regulation of Dlx 5 and its phosphorylation by p38. $\mathrm{J}$ Biol Chem 2008; 283: 3816-3826.

39. Kim Y, Lee M, Wozney JM, Cho J, Ryoo H. BMP2-induced alkaline phosphatase expression is regulated by Dlx5, which is interrupted by Msx2 as a binding competition to the common response element. J Bone Miner Res 2004; 19 : S264-S264.

40. Benson MD, Bargeon JL, Xiao G, Thomas PE, Kim A, Cui $Y$ et al. Identification of a homeodomain binding element in the bone sialoprotein gene promoter that is required for its osteoblast-selective expression. J Biol Chem 2000; 275: 13907-13917.

41. Nakayama $\mathrm{Y}$, Nakajima $\mathrm{Y}$, Kato $\mathrm{N}$, Takai H, Kim DS, Arai M et al. Insulin-like growth factor-1 increases bone sialoprotein (BSP) expression through fibroblast growth factor-2 response element and homeodomain protein-binding site in the proximal promoter of the BSP gene. J Cell Physiol 2006; 208: 326-335.

42. Hayashibara T, Hiraga T, Sugita A, Wang L, Hata K, Ooshima $T$ et al. Regulation of osteoclast differentiation and function by phosphate: potential role of osteoclasts in the skeletal abnormalities in hypophosphatemic conditions. J Bone Miner Res 2007; 22: 1743-1751.

43. Kramer I, Halleux C, Keller H, Pegurri M, Gooi JH, Weber PB et al. Osteocyte Wnt/beta-catenin signaling is required for normal bone homeostasis. Mol Cell Biol 2010; 30: $3071-3085$.

44. Kulkarni RN, Bakker AD, Everts V, Klein-Nulend J. Inhibition of osteoclastogenesis by mechanically loaded osteocytes: involvement of MEPE. Calcif Tissue Int 2010; 87: $461-468$.

45. Liu JC, Lengner CJ, Gaur T, Lou Y, Hussain S, Jones MD et al. Runx2 protein expression utilizes the Runx2 P1 promoter to establish osteoprogenitor cell number for normal bone formation. J Biol Chem 2011; 286: 30057-30070.

46. Aioub M, Lezot F, Molla M, Castaneda B, Robert B, Goubin G et al. Msx2 - / - transgenic mice develop compound amelogenesis imperfecta, dentinogenesis imperfecta and periodental osteopetrosis. Bone 2007; 41: 851-859.

47. Grcevic D, Pejda S, Matthews BG, Repic D, Wang L, Li $\mathrm{H}$ et al. In vivo fate mapping identifies mesenchymal progenitor cells. Stem Cells 2012; 30: 187-196.

48. Hwang J, Kita R, Kwon HS, Choi EH, Lee SH, Udey MC et al. Epidermal ablation of Dlx3 is linked to IL-17-associated skin inflammation. Proc Natl Acad Sci USA 2011; 108: 11566-11571.

\section{Supplementary Information accompanies this paper on Cell Death and Differentiation website (http://www.nature.com/cdd)}

